\title{
Bridging the gaps: getting evidence into practice
}

\author{
William McGuire MD MBChB, Peter W. Fowlie MBChB MSc
}

Previously published at www.cmaj.ca

$\infty \infty$

See related research article by Lee and colleagues, Page 469

$\mathrm{D}$ uring the early 1990s, adoption of key evidencebased interventions for the care of very preterm ( $<32$ weeks' gestation) neonates substantially improved outcomes in this group. In particular, the use of antenatal corticosteroid therapy and postnatal surfactant replacement therapy dramatically reduced early mortality due to respiratory failure. ${ }^{1}$ However, several major morbidities associated with the need for these infants to receive prolonged intensive care remain. These include nosocomial infection and bronchopulmonary dysplasia (chronic respiratory distress with oxygen dependency beyond 36 weeks' postmenstrual age). Because these morbidities are associated with higher risks of mortality and neurodisability, reducing their incidence is a priority for clinicians and care givers. ${ }^{2,3}$

Although several evidence-based interventions for preventing these types of important morbidities exist, these may not be consistently adopted as policy and implemented in practice. ${ }^{4}$ Benchmarking and audit studies in neonatal networks have reveal marked variation in practice even when good evidence exists for specific interventions. ${ }^{5.6}$ Various quality-improvement strategies are available for encouraging clinicians to increase their use of evidence-based practices. A systematic overview suggests that passive dissemination of information, such as distribution of educational materials or didactic lectures, is generally ineffective in driving change. ${ }^{7}$ Multifaceted interventions that act on different levels of barriers to change are more likely to achieve improvements in policy and practice (Box 1).

The Evidence-based Practice for Improving Quality (EPIQ) method, described in the study in this issue by Lee and colleagues, involves the use of a multifaceted qualityimprovement package to reduce nosocomial infection and bronchopulmonary dysplasia rates in preterm infants. ${ }^{8}$ In this cluster randomized controlled trial, participating neonatal centres in Canada were randomly assigned to target reducing either nosocomial infections (infection group) or bronchopulmonary dysplasia (pulmonary group).

During the baseline assessment and training period, clinicians engaged in a process that included training in the principles of continuous quality improvement and systematic appraisal. Following this, a program of rapid change cycles was used to introduce evidence-based interventions. The specific interventions were selected from a menu of options to allow the individual centres to choose the most appropriate interventions based on audit and benchmarking findings supplied by the network (Box 2).

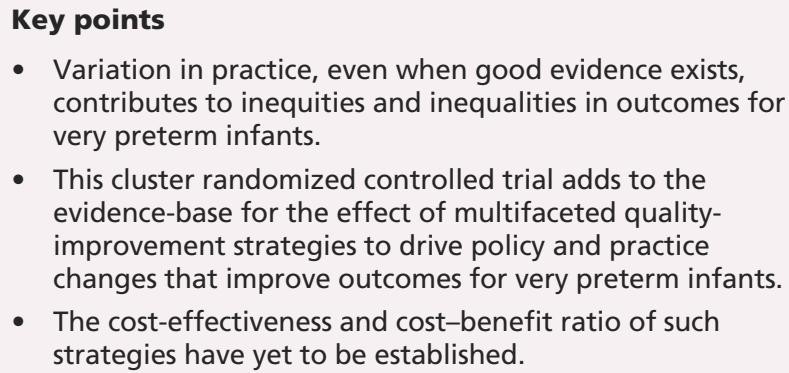

- Variation in practice, even when good evidence exists, contributes to inequities and inequalities in outcomes for very preterm infants.

- This cluster randomized controlled trial adds to the evidence-base for the effect of multifaceted qualityimprovement strategies to drive policy and practice changes that improve outcomes for very preterm infants.

- The cost-effectiveness and cost-benefit ratio of such strategies have yet to be established.

After 2 years, the reduction in the incidence of bronchopulmonary dysplasia from baseline was significantly greater in the pulmonary group than in the infection group. There was no effect on the incidence of nosocomial infection.

Two similar trials have been reported from other large networks in North America. In the late 1990s, a cluster randomized controlled trial by the Vermont-Oxford Network found that a multifaceted quality-improvement strategy increased the rate of early surfactant use. ${ }^{9}$ However, this trial was not designed or powered to asses the effect on the incidence of bronchopulmonary dysplasia. A more recent cluster randomized controlled trial, undertaken by the Neonatal Research Network of the National Institute of Child Health and Development, did not find evidence of an effect on the incidence of bronchopulmonary dysplasia. ${ }^{10}$ This study was similar in design to the study by Lee and colleagues in that individual centres selected which specific interventions to implement. Unlike in the units participating in the current trial, the early administration of surfactant was already an established intervention in most of the participating centres in the Neonatal Research Network. To show the effect of a qualityimprovement initiative on policy and practice, the major evidence-based interventions that are being promoted and adopted must not already be widely used.

The widespread use of the infection-control interventions included in the EPIQ method may be the cause of the failure to detect an effect of the interventions on the incidence of nosocomial infection. The interventions that were most likely

From the Centre for Reviews and Dissemination (McGuire), Hull York Medical School, York; and the Neonatal Intensive Care Unit (Fowlie), Ninewells Hospital and Medical School, Dundee, UK

Cite as CMAJ 2009. DOI:10.1503/cmaj.091243 
Box 1: Key quality-improvement interventions ${ }^{7}$

- Audit and feedback (especially when baseline adherence to recommended practice is low)

- Interactive educational meetings

- Educational outreach by experts or local opinion leaders

- Use of manual or computerized reminders

- Inclusion of local clinicians to achieve a consensus about clinical problems and appropriate responses

to have had an effect, such as aseptic management of central vascular lines and restrictive prescribing of antibiotics, were already standard practice in most centres before the qualityimprovement initiative was started.

Interestingly, clinicians in units in both the EPIQ and the National Institute of Child Health and Development trials chose to adopt a policy of aiming for lower oxygen saturation targets to reduce the incidence of bronchopulmonary dysplasia, despite the fact that this seems to have preempted the availability of robust evidence of benefit. ${ }^{11}$ In contrast, prophylactic caffeine therapy was not a selected strategy because, when the EPIQ trial was started, this was not an established standard of care. Since then, the Canadian-led Caffeine for Apnea of Prematurity trial has shown that prophylactic caffeine reduces the incidence of bronchopulmonary dysplasia and has substantial long-term beneficial effects for very preterm infants. ${ }^{12}$ Given the participation of many neonatal centres worldwide in the Caffeine for Apnea of Prematurity trial, it may be instructive to audit the adoption of prophylactic caffeine into routine care even in the absence of an active dissemination effort. Trial participation itself may be a very effective quality-improvement driver.

Managed clinical networks of neonatal units aim to ensure equity of access to evidence-based interventions. When audit and benchmarking identify practice variations and inequalities in outcomes, an ethical imperative exists to improve these quality markers. The EPIQ study shows the feasibility of applying the "best test," the cluster randomized controlled trial, to evaluate quality-improvement strategies before adopting them as standard practice across networks. The study adds to the accumulating evidence that multifaceted interventions may change practice and outcomes in neonatal intensive care settings. Further evaluations of this type of intervention in a variety of health care settings are needed. As well as showing the effectiveness of a given implementation package, future studies should aim to provide clinically meaningful estimates of effect size by use of something akin to the "number needed to treat" measure now familiar in reports of other interventions. These data can then inform cost-benefit analyses to ensure that limited resources are well used to benefit infants and families and that they could not be better used elsewhere.

\section{Competing interests: None declared.}

Contributors: Both authors jointly wrote the commentary, revised it critically and approved the final version submitted for publication.
Box 2: Interventions introduced by more than 3 centres in the EPIQ trial

Centres randomized to reduce bronchopulmonary dysplasia

- Use of prophylactic surfactants (infants $<1250 \mathrm{~g}$ or $<28$ weeks' gestation)

- Restrict hand ventilation (reduce unmonitored pressure delivery)

- Adopt normocarbia policy

- Target oxygen saturation levels at $88 \%-92 \%$ (alarm limits $85 \%-95 \%$ )

- Use of early parenteral nutrition

Centres randomized to reduce nosocomial infections

- Strategic placement of cleanser dispensers in the neonatal intensive care units and at entrances

- Routine daily chart and audit of skin breaks

- Restrict the number of skin breaks per patient

- Stop the use of antibiotics within 36-48 hours if microbiological cultures are negative

\section{REFERENCES}

1. Fanaroff AA, Hack M, Walsh MC. The NICHD neonatal research network: changes in practice and outcomes during the first 15 years. Semin Perinatol 2003;27:281-7.

2. Stoll BJ, Hansen N. Infections in VLBW infants: studies from the NICHD Neonatal Research Network. Semin Perinatol 2003;27:293-301.

3. Bhandari A, Bhandari V. Pitfalls, problems, and progress in bronchopulmonary dysplasia. Pediatrics 2009;123:1562-73.

4. Brok J, Greisen G, Madsen LP, et al. Agreement between Cochrane Neonatal reviews and clinical practice guidelines for newborns in Denmark: a cross-sectional study. Arch Dis Child Fetal Neonatal Ed 2008;93:F225-9.

5. Horbar JD, Carpenter JH, Buzas J, et al. Timing of initial surfactant treatment for infants 23 to 29 weeks' gestation: Is routine practice evidence based? Pediatrics 2004; 113:1593-602.

6. Lee SK, McMillan DD, Ohlsson A, et al. Variations in practice and outcomes in the Canadian NICU network: 1996-1997. Pediatrics 2000;106:1070-9.

7. Grimshaw J, McAuley LM, Bero LA, et al. Systematic reviews of the effectiveness of quality improvement strategies and programmes. Qual Saf Health Care 2003;12:298-303.

8. Lee SK, Aziz K, Singhal N, et al. Improving the quality of care for infants: a cluster randomized controlled trial. CMAJ 2009. DOI:10.1503/cmaj.081727.

9. Horbar JD, Carpenter JH, Buzas J, et al. Collaborative quality improvement to promote evidence-based surfactant therapy: a cluster randomised trial. BMJ 2004;329:1004-7.

10. Walsh M, Laptook A, Kazzi SN, et al. A cluster randomized trial of benchmarking and multimodal quality improvement to improve survival free of bronchopulmonary dysplasia in infants < 1250 grams birthweight. Pediatrics 2007;119:876-90.

11. Askie LM, Henderson-Smart DJ. Restricted versus liberal oxygen exposure for preventing morbidity and mortality in preterm or low birth weight infants [review]. Cochrane Database Syst Rev 2001;[4]:CD001077.

12. Schmidt B, Roberts RS, Davis P, et al. Long-term effects of caffeine therapy for apnea of prematurity. N Engl J Med 2007;357:1893-902.

Correspondence to: Dr. William McGuire, Centre for Reviews and Dissemination, Hull York Medical School, University of York,

YO10 5DD, UK; william.mcguire@hyms.ac.uk 